\title{
Unitary transformations of a family of two-dimensional anharmonic oscillators
}

\author{
Francisco M Fernández and Javier Garcia \\ INIFTA (UNLP, CCT La Plata-CONICET), División Química Teórica, Blvd. 113 \\ S/N, Sucursal 4, Casilla de Correo 16, 1900 La Plata, Argentina \\ E-mail: fernande@quimica.unlp.edu.ar
}




\begin{abstract}
In this paper we analyze a recent application of perturbation theory by the moment method to a family of two-dimensional anharmonic oscillators. By means of straightforward unitary transformations we show that two of the models studied by the authors are separable. Other is unbounded from below and therefore cannot be successfully treated by perturbation theory unless a complex harmonic frequency is introduced in the renormalization process. We calculate the lowest resonance by means of complex-coordinate rotation and compare its real part with the eigenvalue estimated by the authors. A pair of the remaining oscillators are equivalent as they can be transformed into one another by unitary transformations.
\end{abstract}

\title{
1. Introduction
}

Witwit and Killingbeck [1] applied the perturbation theory by the moment method developed by Fernández and Castro [2-4] to a family two-dimensional anharmonic oscillators with "mixed parity potentials". By means of renormalization of the perturbation series they obtained reasonably accurate eigenvalues for a particular set of potential parameters. The authors did not take into account the symmetry of the perturbation which had proved to be extremely useful in the treatment of similar quantum-mechanical models [5, 6]. Lately, the application of point group symmetry (PGS) has proved to be suitable for the analysis of a variety of non-Hermitian anharmonic oscillators [7-10].

An attempt to apply PGS to the anharmonic oscillators studied by Witwit and Killingbeck revealed some interesting facts that we want to discuss in this paper. In section 2 we briefly summarize some aspects of unitary transformations and point groups that will be useful in the subsequent sections. In section 3 we apply those concepts to the anharmonic oscillators studied by Witwit and Killingbeck. Finally, in section 4 we summarize the main results of this paper and draw conclusions. 


\section{Unitary transformations and point group}

If $U$ is an invertible operator, then the Hamiltonian operators $H$ and $\tilde{H}=U H U^{-1}$ are isospectral. In this paper we are interested only in unitary transformations $U^{-1}=U^{\dagger}$, where $U^{\dagger}$ is the adjoint of $U$.

The set of unitary transformations $U_{i}, i=1,2, \ldots, h$ that leave a given Hamiltonian operator $H$ invariant $U_{i} H U_{i}^{\dagger}=H$ form a group with respect to the composition $U_{i} U_{j}$ [11, 12]. It follows from the invariance of $H$ that $\left[H, U_{i}\right]=0$. Clearly, if $\psi$ is an eigenfunction of $H$ with eigenvalue $E$ then $U_{i} \psi$ is also eigenfunction with the same eigenvalue as follows from $H U_{i} \psi=U_{i} H \psi=E U_{i} \psi$. The eigenfunctions of $H$ are bases for the irreducible representations (irreps) of the point group $G$ of $H$ and can therefore be classified according to them [11,12.

If $G=\left\{U_{i}, i=1,2, \ldots, h\right\}$ is the point group for $H$ and $\tilde{H}=U H U^{\dagger}$ then $\tilde{G}=\left\{\tilde{U}_{i}=U U_{i} U^{\dagger}, i=1,2, \ldots, h\right\}$ is the point group for $\tilde{H}$. Both groups are isomorphic as follows from $\tilde{U}_{i} \tilde{U}_{j}=U U_{i} U_{j} U^{\dagger}$. It the following section we apply these simple well known results to the anharmonic oscillators studied by Witwit and Killingbeck.

\section{The anharmonic oscillators}

Witwit and Killingbeck [1] applied perturbation theory by the moment method (which they baptized inner product method) to anharmonic oscillators of the form

$$
\begin{aligned}
& H \quad=p_{x}^{2}+p_{y}^{2}+x^{2}+y^{2}+\lambda V(x, y) \\
& V(x, y)=a_{x x} x^{4}+4 b_{x y} x^{3} y+6 c_{x y} x^{2} y^{2}+4 b_{y x} x y^{3}+a_{y y} y^{4}
\end{aligned}
$$

where $\lambda$ is the perturbation parameter. They chose a few different sets of potential parameters that we analyze in what follows:

Case 1: $a_{x x}=b_{x y}=c_{x y}=b_{y x}=a_{y y}=1$. It leads to

$$
H=p_{x}^{2}+p_{y}^{2}+x^{2}+y^{2}+\lambda\left(x^{4}+4 x^{3} y+6 x^{2} y^{2}+4 x y^{3}+y^{4}\right) \text {. }
$$


The unitary transformation

$$
U:(x, y) \rightarrow\left(\frac{x}{\sqrt{2}}-\frac{y}{\sqrt{2}},-\frac{x}{\sqrt{2}}-\frac{y}{\sqrt{2}}\right),
$$

decouples the degrees of freedom and leads to

$$
\tilde{H}=p_{x}^{2}+p_{y}^{2}+x^{2}+y^{2}+4 \lambda y^{4},
$$

facilitating the calculation enormously. For instance, a straightforward application of the Riccati-Padé method (RPM) [13, 14] for $\lambda=1$ yields

$$
E_{0}^{C 1}=2.903136945459000022293850722201023931817
$$

that considerably improves the estimate of Witwit and Killingbeck [1].

Case 2: $a_{x x}=c_{x y}=a_{y y}=1, b_{x y}=b_{y x}=0$. This problem

$$
H=p_{x}^{2}+p_{y}^{2}+x^{2}+y^{2}+\lambda\left(x^{4}+6 x^{2} y^{2}+y^{4}\right)
$$

is also separable by means of the unitary transformation

$$
U:(x, y) \rightarrow\left(\frac{x}{\sqrt{2}}+\frac{y}{\sqrt{2}}, \frac{y}{\sqrt{2}}-\frac{x}{\sqrt{2}}\right)
$$

that leads to

$$
\tilde{H}=p_{x}^{2}+p_{y}^{2}+x^{2}+y^{2}+2 \lambda\left(x^{4}+y^{4}\right)
$$

The RPM for the ground state of this problems with $\lambda=10^{6}$ yields

$$
E_{0}^{C 2}=267.2002503791361424618416691534920465128
$$

which also improves the result reported by those authors.

Case 3: $a_{x x}=a_{y y}=0, c_{x y}=b_{x y}=b_{y x}=1$. The resulting anharmonic oscillator

$$
H=p_{x}^{2}+p_{y}^{2}+x^{2}+y^{2}+\lambda\left(4 x^{3} y+6 x^{2} y^{2}+4 x y^{3}\right)
$$

is obviously unbounded from below and does not support bound states. For this reason the results of Witwit and Killingbeck [1] for this example were considerably less accurate than for the other ones. They did not mention this fact and did not report results for $\lambda>0.12$, probably because the increasingly greater imaginary part of the resonances made the straightforward perturbation calculation unreliable. In principle, 
one can obtain a convergent perturbation series if the harmonic frequency introduced for renormalization is allowed to be complex instead of being restricted to real values.

This problem exhibits symmetry $C_{2 v}$ and the same change of variables shown above in equation (7) leads to

$$
\tilde{H}=p_{x}^{2}+p_{y}^{2}+x^{2}+y^{2}+\lambda\left(\frac{7 y^{4}}{2}-3 x^{2} y^{2}-\frac{x^{4}}{2}\right),
$$

with obviously the same symmetry $C_{2 v}$. However, since this potential exhibits only even powers of the variables the authors could have simplified the calculation by resorting to the simple symmetry analysis already used in earlier papers [15,16]. For a more rigorous discussion of the interplay between symmetry and perturbation theory by the moment method for this kind of anharmonic oscillators see a recent paper by Fernández [17].

We calculated the lowest resonance for the anharmonic oscillator (10) by means of the complex rotation method [18 21] using finite basis sets of eigenfunctions of $H_{0}$ of increasing dimension up to $30^{2} \times 30^{2}$ and a roughly optimal rotation angle $\theta=0.06 \pi$. Table 1 shows present results and those of Witwit and Killingbeck [1] for some values of $\lambda$. Note that the error in the eigenvalue estimated by those authors is of the order of $|\Im E|$ that increases with $\lambda$ as argued above. We have chosen the greatest values of $\lambda$ considered by those authors in order to illustrate this point more clearly.

Case 4: $a_{x x}=a_{y y}=c_{x y}=1, b_{x y}=b_{y x}=-1$. The inclusion of this case in the discussion is surprising because the resulting Hamiltonian becomes the one for Case 1 by means of the unitary transformations $U:(x, y) \rightarrow(-x, y)$ or $U:(x, y) \rightarrow(x,-y)$. Note that the authors obtained exactly the same eigenvalues with the same accuracy for both cases as expected. There is no point in discussing this case here.

Case 5: $c_{x y}=1, a_{x x}=a_{y y}=b_{x y}=b_{y x}=0$. The resulting Hamiltonian

$$
H=p_{x}^{2}+p_{y}^{2}+x^{2}+y^{2}+6 \lambda x^{2} y^{2}
$$

does not exhibit "mixed parity" but symmetry $C_{4 v}$ [5] and has been chosen as benchmark many times in the past (other references are given elsewhere [22]) even for an earlier application of perturbation theory by the moment method [15, 16]. 


\section{Conclusions}

The aim of the addendum by Witwit and Killingbeck [1] was the application of the perturbation theory by the moment method to two-dimensional oscillators with mixedparity potentials; that is to say: with even and odd powers of the variables $x$ and $y$. However, present analysis shows that two of the models studied by those authors (Case 1 and Case 2) are trivial in the sense that they are separable by unitary transformations.

What is more, the resulting Hamiltonians exhibit only even powers of the coordinates. The Hamiltonian operator for Case 3 is unbounded from below and therefore cannot be successfully treated by perturbation theory unless a complex harmonic frequency is introduced in the renormalization process. Besides, this Hamiltonian can be transformed into one with only even powers of the coordinates. The Hamiltonian for Case 4 is trivially isospectral to the one for Case 1 and, therefore, does not add anything relevant to the discussion. Finally, the Hamiltonian for Case 5 does not exhibit mixed parity and was treated before by the same authors [15,16] (see Fernández [17] for a discussion based on PGS).

\section{Acknowledgments}

This report has been financially supported by PIP No. 11420110100062 (Consejo Nacional de Investigaciones Cientificas y Tecnicas, República Argentina)

[1] Witwit N R M and Killingbeck J 1993 J. Phys. A 263659.

[2] Fernández F M and Castro E A 1984 Int. J. Quantum Chem. 26497.

[3] Arteca G A, Fernández F M, Mesón A M, and Castro E A 1984 Physica A 128253.

[4] Fernández F M and Castro E A 1985 Int. J. Quantum Chem. 28603.

[5] Pullen R A and Edmonds A R 1981 J. Phys. A 14 L477.

[6] Pullen R A and Edmonds A R 1981 J. Phys. A 14 L319.

[7] Fernández F M and Garcia J 2014 Ann. Phys. 342195.

[8] Fernández F M and Garcia J 2014 J. Math. Phys. 55042107.

[9] Amore P, Fernández F M, and Garcia J 2014 Ann. Phys., in the press. 
[10] Amore P, Fernández F M, and Garcia J 2014 Non-Hermitian oscillators with Td symmetry arXiv:1409.2672 [quant-ph]

[11] Tinkham M 1964 Group Theory and Quantum Mechanics (McGraw-Hill Book Company, New York).

[12] Cotton F A 1990 Chemical Applications of Group Theory (John Wiley \& Sons, New York).

[13] Fernández F M, Ma Q, and Tipping R H 1989 Phys. Rev. A 406149.

[14] Fernández F M, Ma Q, DeSmet D J, and Tipping R H 1989 Can. J. Phys. 67931.

[15] Killingbeck J and Jones M N 1986 J. Phys. A 19705.

[16] Witwit N R M 1991 J. Phys. A 244535.

[17] Fernández F M 2014 Perturbation theory by the moment method and point-group symmetry. arXiv:1409.4120 [quant-ph].

[18] Balslev E and Combes J C 1971 Commun. Math. Phys. 22280.

[19] Reinhardt W P 1976 Int. J. Quantum Chem. Symposium 10359.

[20] Chu S-I and Reinhardt W P 1977 Phys. Rev. Lett. 391195.

[21] Yaris R, Bendler J, Lovett R A, Bender C A, and Fedders P A 1978 Phys. Rev. A 181816.

[22] Amore P and Fernández F M 2010 Phys. Scr. 81045011. 
Table 1. Lowest resonance for the anharmonic oscillator (10)

\begin{tabular}{llcl}
\hline$\lambda$ & Ref. [1] & $\Re E$ & $\Im E$ \\
\hline 0.10 & 2.0733 & 2.07335064 & -0.000459014 \\
0.12 & 2.08 & 2.0746525 & -0.0022857 \\
0.13 & & 2.0738983 & -0.0041665 \\
0.14 & & 2.0724187 & -0.0068909 \\
\hline
\end{tabular}

